\title{
Índice de función psicológica en preescolares de distintos estratos socioeconómicos: un estudio piloto
}

\author{
Ángel Alberto Puig Lagunes \\ anpuig@uv.mx \\ https://orcid.org/0000-0003-0177-3921 \\ Doctor en Investigaciones cerebrales, académico \\ de la Facultad de Medicina, Universidad Veracruzana, \\ campus Minatitlán, Veracruz, México. \\ Martha Elia Yannine Bautista Torres \\ mabautista@uv.mx \\ https://orcid.org/0000-0001-8939-0196 \\ Maestra en ciencias, académica \\ de la Facultad de Medicina, Universidad Veracruzana, \\ campus Minatitlán, Veracruz, México. \\ María Fernanda Zapata de la Rosa \\ zS18018462@estudiantes.uv.mx \\ https://orcid.org/0000-0002-3142-9956 \\ Estudiante de la Facultad de Medicina, \\ Universidad Veracruzana, campus Minatitlán, \\ Veracruz, México. \\ Brenda Itzel Molina Cadena \\ zS18002477@estudiantes.uv.mx \\ https://orcid.org/0000-0002-2519-0432 \\ Estudiante de la Facultad de Medicina, \\ Universidad Veracruzana, campus Minatitlán, \\ Veracruz, México. \\ Angel Puig Nolasco \\ apuign@hotnail.com \\ https://orcid.org/0000-0003-4876-9727 \\ Doctor en Neuroetología, académico \\ de la Facultad de Medicina, Universidad Veracruzana, \\ campus Minatitlán, Veracruz, México.
}

\section{RESUMEN}

Introducción: Durante los primeros cinco años de vida, el cerebro en desarrollo es más sensible a la estimulación, crianza y otros factores que pueden intervenir positiva y negativamente su desarrollo. Objetivo: Determinar el Índice de Función Psicológica 
(IFP) en preescolares de diferentes estratos socioeconómicos y relacionarlo con sus características sociodemográficas y estilos de vida. Métodos: Se determinó el IFP a través de la prueba de dibujo de la figura humana de Goodenough en 792 preescolares de instituciones públicas y privadas de diferentes estratos socioeconómicos; además, se identificaron sus datos sociodemográficos y de estilo de vida por medio de un cuestionario. Resultados: $24,6 \%$ de los preescolares manifestaron un IFP inferior al promedio, 63,6\% estuvieron alrededor del promedio y 11,7\% fueron superiores al promedio. Los preescolares del estrato socioeconómico medio mostraron mayor puntuación. La edad, el sexo y el grado escolar estuvieron en asociación con el IFP. Conclusiones: En la población estudiada, uno de cada cuatro preescolares muestra un IFP inferior al promedio, el IFP es mayor en preescolares de estrato medio. Se necesita implementar estrategias y prácticas más benéficas dentro del hogar y de las escuelas, que estimulen el desarrollo de la motricidad gruesa y de las funciones mentales superiores de los preescolares.

Palabras clave: prueba psicológica; psicología educacional; psicología pediátrica; trastornos del desarrollo infantil; trastornos de retraso en el desarrollo. 


\title{
Psychological function index in preschoolers of different socioeconomic strata: a pilot study
}

\begin{abstract}
Introduction: During the first five years of life, the developing brain is more sensitive to stimulation and nurturing, just like other factors that intervene positively and negatively in its development. Objective: To determine Psychological function index (PFI) in preschoolers from different socioeconomic strata and relate it to sociodemographic and lifestyle characteristics. Methods: the PFI was determined through Goodenough DrawA-Person in 792 preschoolers from different socioeconomic strata from public and private educational institutions, also the main sociodemographic and lifestyle data were identified. Results: $24,6 \%$ of preschoolers showed PFI below average, 63,6\% demonstrated a PFI around the average, and 11,7\% higher than the average. A higher score was observed in the Middle Socioeconomic Strata followed by the high stratum and low stratum. On the other hand, age, sex and school grade were in association with PFI. Conclusions: The present exploratory study showed that one in four preschoolers showed a lower than average PFI, the PFI is higher in middle-class preschoolers. In addition, strategies need to be implemented to develop more beneficial practices within the home and in schools, which can stimulate the development of gross motor skills and higher mental functions in preschoolers.
\end{abstract}

Keywords: psychological testing; educational psychology; pediatric psychology; developmental disorders; developmental retardation disorders.

Artículo recibido: 30 noviembre. 2021 Aceptado para publicación: 29 diciembre 2021

Correspondencia: anpuig@uv.mx Conflictos de Interés: Ninguna que declarar 


\section{INTRODUCCIÓN}

Durante los primeros cinco años de vida, el ser humano se encuentra en un período crítico de cambios en la estructura cerebral, crecimiento y neurodesarrollo; simultáneamente, el individuo está expuesto a factores biológicos y psicosociales que influencian ambos fenómenos tanto positiva como negativamente (Lebel, \& Deoni, 2018; Mamani-Ortiz, et $a l ., 2014)$. Diversos estudios consideran que esta etapa de la infancia precoz impacta de manera significativa en la calidad de vida, salud y bienestar, incluso en la adultez y que todos estos son el resultado de un progreso adecuado del crecimiento y desarrollo (Engle, et al., 2007).

El estrato socioeconómico, la estimulación neurosensorial, la educación, la interacción padres-hijos, el estado nutricional, el estado preconcepcional, el estado de salud físico y mental, así como el contexto social, escolar y familiar se asocian con la evolución del crecimiento y desarrollo infantil; algunos estudios consideran que otros factores, como los programas y políticas empleadas en la infancia temprana, influyen positivamente en el desarrollo neurológico temprano y que intervenciones en preescolares reducen el impacto de los factores de riesgo, las inequidades en la infancia e incrementan la productividad (Noble, et al., 2015; Black, et al., 2017; Herrera-Mora et al., 2019; Daelmans, et al., 2017).

La vigilancia y monitoreo del desarrollo cognitivo, permite establecer sospechas diagnósticas precoces y oportunas de niños con alto riesgo de presentar una alteración del desarrollo como discapacidad intelectual, desórdenes de la comunicación, problemas de aprendizaje, de la percepción visual y motores, así como de su tratamiento temprano y la generación de intervenciones adecuadas (Zeegers, et al., 2009; Hamilton, 2006). Para medir el desempeño cognitivo en niños de 4 a 10 años se utiliza el Índice de Función Psicológica (IFP), a través de una estimación numérica, que valora el neurodesarrollo de acuerdo a diferentes áreas y competencias propias del infante (Springer, et al., 2020). En 2020, la Organización Mundial de la Salud (OMS) junto con el Fondo de las Naciones Unidas para la Infancia (UNICEF) y the Lancet publicaron un informe que documentaba que, a nivel global, alrededor de 250 millones de niños menores de 5 años de países de ingresos bajos y medios corrían el riesgo de no alcanzar su potencial de desarrollo (Clark, et al., 2020). Sin embargo, en algunos países de América Latina y el Caribe estas cifras 
aumentan alrededor del $15 \%$, planteando problemas económicos, médicos y psicológicos (Vitrikas, et al., 2017).

Algunas pruebas psicométricas o proyectivas presentan grandes limitaciones debido a la complejidad de su aplicación y a los altos costos económicos, sociales y temporales, presentes especialmente en países en vías de desarrollo, donde el déficit de recursos es una barrera para su aplicación (Gridley, et al., 2019). El uso de pruebas gratuitas y de fácil aplicación, cuyos resultados se consideran detecciones preliminares de infantes en riesgo, permiten su utilización como prueba de tamizaje (Springer et al., 2020; SánchezVillena, \& Ventura-León, 2020; Gutiérrez-Duarte, \& Ruiz-León, 2018). La prueba del Dibujo de la Figura Humana de Goodenough-Harris (DFH), es una herramienta psicométrica de tipo proyectiva que permite el análisis sistemático de los componentes un dibujo elaborado por el niño evaluado; a través de un sistema de puntaje, se estima la madurez conceptual y cognitiva del infante que permite determinar el IFP (El-Shafie, et al., 2019) ${ }^{16}$. La prueba DFH se considera de fácil aplicación, costo efectivo, práctica, de corta duración y universal, debido a que la imagen de una figura humana no se ve afectada por la cultura o experiencias educativas del infante; esto la convierte en una opción ampliamente aceptada en la actualidad (Baraheni, et al., 2018). Diversos estudios, validan al DFH como una herramienta útil para determinar el nivel de desarrollo de las habilidades visoconstructivas de niños menores de 8 años (Springer, et al., 2020; ElShafie, et al., 2019; Baraheni, et al., 2018; Rinaldi, \& Alves, 2014).

En México, se han analizado pocos estudios que evalúen el desarrollo en escolares sanos; un estudio realizado en niños Indígenas Yaquis de segundo y tercer grado de primaria, mostró que $18 \%$ de estos tienen puntajes bajos en la prueba de dos Figuras Humanas, mientras que en la Escala Wechsler de Inteligencia para Niños-IV, 57 \% mostraron puntajes bajos (Fernández-Nistal, et al., 2018). Por otro lado, por medio de los Indicadores de Riesgo del Perfil de Conductas de Desarrollo-Revisados (INDIPCD-R), aplicado en niños de 2 a 4 años se observó que $26 \%$ de los infantes tuvo más de 11 indicadores de riesgo de retrasos y alteraciones del desarrollo (Bolaños, et al., 2015).

Por tales motivos, debido a la carencia de información a nivel nacional e internacional principalmente en América Latina y nula información en la región referente al desarrollo en preescolares, y las posibles variables que intervienen positiva o negativamente en su desarrollo, asimismo, considerando a las pruebas proyectivas como una herramienta de 
cribado preliminar en la determinación de poblaciones infantiles en riesgo, para contribuir con un seguimiento adecuado y disminuir el impacto en su desarrollo intelectual y social, el objetivo de este estudio fue determinar el IFP en preescolares de diferentes estratos socioeconómicos, así cómo su asociación con características sociodemográficas y de estilos de vida.

\section{METODOLOGÍA}

Se realizó un estudio exploratorio, transversal, descriptivo, analítico y correlacional en preescolares de instituciones educativas públicas y privadas de diferentes estratos socioeconómicos, pertenecientes a los municipios de Minatitlán y Zaragoza, Veracruz, entre septiembre y noviembre del 2018. El diseño del estudio fue revisado y aprobado por el Comité de Bioética e investigación Institucional de la Facultad de Medicina, Universidad Veracruzana, campus Minatitlán (folio CIE 003-2018).

Se realizó una solicitud a los directivos y padres de familia de las instituciones educativas para la participación voluntariamente en el estudio piloto, informándoles sobre el objetivo del proyecto, los procedimientos de aplicación del cuestionario socioeconómico y la prueba de DFH. Los criterios de inclusión fueron: estar inscritos en el periodo seleccionado y cursando la educación preescolar, tener una agudeza visual y auditiva normal o corregida, firmar el consentimiento informado, contestar en su totalidad el cuestionario socioeconómico y realizar el DFH. Los criterios de exclusión fueron: tener diagnóstico previo de Retraso global del desarrollo (RGD) o de otros problemas neurológicos o psiquiátricos, presentar limitaciones físicas que impidieran la ejecución de las pruebas.

Se incluyeron nueve instituciones educativas: dos escuelas públicas del municipio de Zaragoza con 222 niños, conformaron el grupo de estrato socioeconómico bajo (ESB), en este municipio, para el 2015 se estimó que 53.3 y $22.8 \%$ de la población se encontraba en pobreza modera y extrema respectivamente (SEFIPLAN, 2018); siete escuelas del municipio de Minatitlán, de las cuales cuatro instituciones públicas del con 470 niños conformando el estrato Bajo-Medio (ESM); y tres escuelas privadas con 184 niños que formaron el estrato Medio-Alto (ESA). En 2015 en el Mpio. Minatitlán se estableció que $37.7 \%$ de la población se encontraba en pobreza modera y $10.1 \%$ en pobreza extrema, sin embargo, estos datos son inferiores en la cabecera municipal donde se realizó el estudio (SEFIPLAN, 2020) . 
Para determinar diferencias entre edades, se aplicó regla de Sturges generando los siguientes grupos etarios: grupo 1: de 2.10 a 3.5 años; 2: de 3.6 a 4.1 años; 3 : de 4.2 a 4.9 años; 4: de 4.10 a 5.5 años y 5: de 5.6 a 6.1 años.

\section{INSTRUMENTOS}

Se aplicó un cuestionario sociodemográfico y de hábitos de vida que contenía: edad, sexo, grado de estudios, orden de nacimiento, nacimiento a término o pretérmino, número de hermanos, hábitos de sueño, práctica de deporte, presencia de mascotas, relación y estructura familiar (parental o mono parental), relación padres-hijos, nivel de estudio de los padres, prácticas recreativas y de ocio en el hogar, y uso de dispositivos electrónicos (tabletas, celulares y televisión).

\section{La prueba del Dibujo de la Figura Humana de Goodenough-Harris (DFH)}

Se evaluó a los preescolares con la prueba de $\mathrm{DFH}$, la cual mide las habilidades cognitivas con una aproximación diagnóstica, permitiendo su utilización como prueba de tamizaje (Springer, et al., 2020; Tükel, et al., 2018; Ramírez Montenegro, et al., 2014; Piotrowski, 2015). Esta prueba se compone de 51 ítems, que se basan en la valoración de rasgos físicos, presentes o ausentes, ilustrados en el dibujo. La puntuación final, permite la estimación del IFP mediante la expresión de la edad mental del preescolar y su comparación con la edad cronológica. La estimación de la edad mental se convierte en un índice estandarizado, por lo tanto, representa una estimación del desarrollo mental de los niños de 3 a 10 años. Se consideró que un total por debajo de 85 puntos representa un puntaje inferior al promedio, de 85 a 119 como un valor promedio y cifras mayores a 120 puntos como un valor superior al promedio (Springer, et al., 2020). Todos los dibujos fueron evaluados por separado por un grupo de psicólogos expertos en el área de acuerdo al sistema de puntuación del DFH.

\section{Procedimiento}

Posterior a la autorización de la realización del estudio dentro de las instituciones educativas, se entregó el consentimiento informado a los padres y/o tutores de los preescolares. Aquellos que aceptaron participar, se les aplicó el cuestionario sociodemográfico y de estilos de vida de los preescolares. La prueba del DFH se aplicó de manera grupal en un ambiente cómodo, sin distracciones y/o estímulos que los distrajese de la ejecución de la actividad, brindando a los niños únicamente las herramientas indispensables para su realización (hoja en blanco y lápiz No. 2). A su vez, 
se indicó que los preescolares dibujasen a una persona completa, enfatizando que los dibujos no debían ser coloreados, ni caricaturizados ni en forma de "palitos". Además, se enfatizó que no debía dar ningún tipo de ayuda como: guiar la mano, decirle si le falta algo a su dibujo, etc., ya que es importante determinar su aprendizaje adaptativo. No hubo tiempo límite, sin embargo, se anotó si alguno ocupo más de 20 minutos.

\section{Análisis estadístico}

Las variables cualitativas se analizaron como frecuencias y porcentajes, mientras que las variables cuantitativas mediante las medias y el error estándar. El análisis bivariado del IFP y las variables sociodemográficas se analizaron mediante la prueba de chi-cuadrada de Pearson. Las comparaciones del IFP entre las instituciones educativas, el grado de estudio, sexo y edades y otras más se efectuaron mediante la aplicación de la prueba de $\mathrm{t}$ de student y Análisis de varianza (ANOVA). Las pruebas se seleccionaron dependiendo la normalidad y homocedasticidad de varianza. Todos los datos se analizaron con el paquete estadístico SPSS versión 19 para macOS (IBM Corp., Armonk, NY, Estados Unidos). Se consideró un nivel de confianza del $95 \%$ en todas las pruebas estadísticas y se consideró como estadísticamente significativo el valor $\mathrm{p}<0,05$.

\section{RESULTADOS}

Se analizó el resultado de 792 preescolares (49 \% niñas y $51 \%$ niños), con una edad promedio de $4.3 \pm 0.87$ años (rango 2 años 10 meses hasta 6 años) de los cuales 204 (25.8 \%) fueron de ESB, 421 (53.2 \%) de ESM, y 167 (21.1\%) de ESA y distribuidos por grado de la siguiente manera: $142(17.9 \%)$ pertenecían a primer grado, $320(40.4 \%)$ a segundo y $330(41.7 \%)$ a tercero.

De manera general, el $24.6 \%$ de los preescolares presentaron un IFP inferior al promedio, $63.6 \%$ mostraron valores promedio y $11.7 \%$ fue superior al promedio (tabla 1). Los preescolares de primero y segundo año mostraron que su edad mental $(3.05 \pm 0.04$ años y $4.1 \pm 0.03$ años, respectivamente) fue menor $(\mathrm{p}=0.0001)$ en comparación con la cronológica ( $3.3 \pm 0.04$ años; $4.3 \pm 0.04$ años, respectivamente). 


\section{Tabla 1}

\begin{tabular}{|c|c|c|c|c|c|}
\hline & Clasificación del CD & $\begin{array}{c}\text { Abajo del } \\
\text { promedio } \\
\text { n (\%) }\end{array}$ & $\begin{array}{l}\text { Promedio } \\
\text { n }(\%)\end{array}$ & $\begin{array}{c}\text { Superior al } \\
\text { promedio } \\
\text { n }(\%)\end{array}$ & Total \\
\hline & General & 195 (24.6) & $504(63.6)$ & $93(11.7)$ & 792 \\
\hline \multirow{3}{*}{$\begin{array}{l}\text { Estrato } \\
\text { socioeconomico }\end{array}$} & ESB & $94(22.3)$ & $272(64.4)$ & $55(13.3)$ & 421 \\
\hline & ESM & $49(25.5)$ & $136(66.6)$ & $19(9.3)$ & 204 \\
\hline & ESA & $52(31.1)$ & $96(57.4)$ & $19(11.3)$ & 167 \\
\hline \multirow{2}{*}{ Sexo } & Femenino & $109(28.1)$ & $240(61.8)$ & $39(10.1)$ & 388 \\
\hline & Masculino* & $86(21.2)$ & $264(65.3)$ & $54(13.3)$ & 404 \\
\hline \multirow{5}{*}{ Edad } & $1^{*}$ & $67(69.8)$ & $20(20.8)$ & $9(9.3)$ & 96 \\
\hline & 2 & $48(28.9)$ & 85 (51.2) & $33(19.8)$ & 166 \\
\hline & 3 & 37 (17.2) & $149(69.3)$ & $29(13.4)$ & 215 \\
\hline & 4 & $21(12.5)$ & $125(78.9)$ & $21(12.5)$ & 167 \\
\hline & 5 & $22(14.8)$ & $125(84.5)$ & $1(0.6)$ & 148 \\
\hline \multirow{3}{*}{ Grado } & $1^{o *}$ & $97(68.3)$ & $37(26.1)$ & $8(5.6)$ & 142 \\
\hline & $2^{o}$ & $66(20.6)$ & $195(61)$ & $59(18.4)$ & 320 \\
\hline & $3^{\circ}$ & $50(15.1)$ & $254(77)$ & $26(7.9)$ & 330 \\
\hline
\end{tabular}

* $p<0.05$, Los valores expresan la frecuencia y porcentaje. Cociente de desarrollo (CD);

Estrato socioeconómico Bajo (ESB), Estrato socioeconómico bajo-medio (ESM), Estrato socioeconómico medio-alto (ESA).

De acuerdo con las variables sociodemográficas, se observaron diferencias significativas entre los grupos etarios (Kruskal-Wallis $=77.6, \mathrm{p}<0.0001$ ), siendo los preescolares del grupo 1 los que obtuvieron menores puntajes del IFP $(88.5 \pm 1.5)$, seguido del grupo 5 $(94.3 \pm 0.8)$, mientras que los demás grupos fueron similares $(100 \pm 0.7)$. Por otro lado, se evidenciaron diferencias del IFP entre género $(\mathrm{t}=2.6, \mathrm{p}=0.0092)$ observándose mayores puntajes en los niños $(99.4 \pm 0.8)$ en comparación con las niñas $(96.4 \pm 0.8)$. Los grados escolares también difirieron (Kruskal-Wallis $=93.6, \mathrm{p}<0.0001$ ), los preescolares de primero exhibieron menor IFP $(86.8 \pm 1)$ en comparación con los de segundo (102.2 \pm 1$)$ y tercer grado $(97.7 \pm 0.7)$. Al comparar el IFP entre los estratos socioeconómicos, se observó mayor puntuación en los de ESM (99.1 \pm 0.8; ANOVA F= 3.430, p=0.032), seguido del ESA y del ESB $(96.4 \pm 1$ y $96.1 \pm 1$ respectivamente), (figura 1). Por 
otro lado, no se observaron diferencias o asociaciones entre el IFP y algunas variables relacionadas con el estilo de vida como: orden de nacimiento, nacimiento a término o pretérmino, número de hermanos, hora de dormir, práctica de algún deporte, convivencia con mascotas, estructura familiar, tiempo dedicado a juego, charlas y salidas recreativas, nivel de estudios de los padres y uso de tabletas, celulares y televisión.

\section{Figura 1}

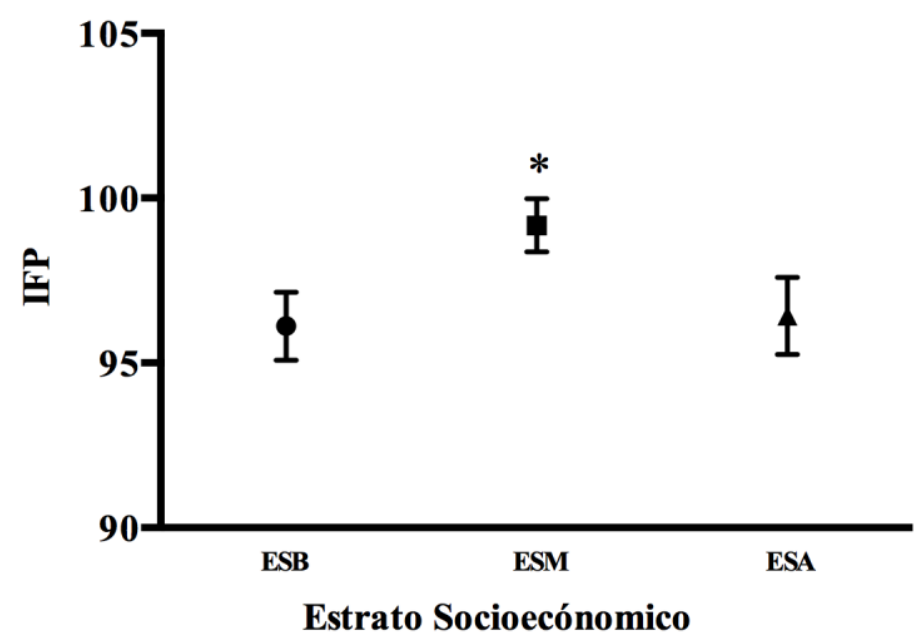

Figura 1.- Índice de función psicológica (IFP) de los preescolares pertenecientes a diferentes estratos socioeconómicos. Estrato socioeconómico Bajo (ESB), Estrato socioeconómico bajo-medio (ESM), Estrato socioeconómico medio-alto (ESA), * $p<0.05$ Al comparar los IFP entre las escuelas de acuerdo con su estrato socioeconómico, se observaron diferencias significativas (Kruskal-Wallis $=25.1, \mathrm{p}=0.0015)$, siendo mayores en los preescolares de una escuela de ESM $(102 \pm 2.4)$ y menores en una escuela de ESB (91.6 \pm 1.6). Al comparar los grados escolares, los de primero de una escuela ESA mostraron menores IFP $(81.7 \pm 0.4)$ en comparación con una de ESM $(91.4 \pm 2.3)$. De manera similar, los de tercero de una escuela ESM, mostraron mayores IFP (105.5 \pm 2.3$)$ en comparación con otras de ESM $(96.3 \pm 1.1)$ y una ESA $(92.7 \pm 3.5)$, (tabla 2). 


\section{Tabla 2}

\begin{tabular}{|c|c|c|c|c|c|c|c|c|c|}
\hline $\begin{array}{l}\text { Estrato socio- } \\
\text { económico }\end{array}$ & \multicolumn{2}{|c|}{ Bajo (ESB) } & \multicolumn{4}{|c|}{ Bajo-Medio (ESM) } & \multicolumn{3}{|c|}{ Medio-alto (ESA) } \\
\hline Escuela (n) & $\begin{array}{c}1 \\
(58)\end{array}$ & $\begin{array}{c}2 \\
(146)\end{array}$ & $\begin{array}{c}3 \\
(56)\end{array}$ & $\begin{array}{c}4 \\
(55)\end{array}$ & $\begin{array}{c}5 \\
(212)\end{array}$ & $\begin{array}{c}6 \\
(98)\end{array}$ & $\begin{array}{c}7 \\
(26)\end{array}$ & $\begin{array}{c}8 \\
(107)\end{array}$ & $\begin{array}{c}9 \\
(34)\end{array}$ \\
\hline General & $90.3 \pm 1$ & $98.4 \pm 1$ & $102 \pm 2 *$ & $97.2 \pm 2$ & $98 \pm 1$ & $101.1 \pm 1$ & $102.3 \pm 4$ & $95.2 \pm 1$ & $97.2 \pm 2$ \\
\hline $1^{\circ}$ grado & $89.2 \pm 5$ & $85 \pm 2$ & $99.2 \pm 6$ & $91.7 \pm 5$ & $\begin{array}{r}91.4 \pm \\
2 *\end{array}$ & $89.1 \pm 3$ & $94.4 . \pm 5$ & $81.7 \pm 0.4$ & $\mathrm{~S} / \mathrm{D}$ \\
\hline $2^{\circ}$ grado & $91.6 \pm 2$ & $102.8 \pm 2$ & $105.7 \pm 6$ & $104.9 \pm 3$ & $\begin{array}{c}102.3 \pm \\
1\end{array}$ & $100.1 \pm 3$ & $117 \pm 15$ & $104.4 \pm 2$ & $101.8 \pm 2$ \\
\hline $3^{\circ}$ grado & $89.8 \pm 2$ & $98.3 \pm 1$ & $102.5 \pm 2$ & $91.2 \pm 1$ & $96.3 \pm 1$ & $\begin{array}{r}105.5 \pm \\
2 *\end{array}$ & $102.1 . \pm 4$ & $94.5 \pm 1$ & $92.7 \pm 3$ \\
\hline
\end{tabular}

$* p<0.05$, Sin datos (S/D). Estrato socioeconómico Bajo (ESB), Estrato socioeconómico bajo-medio (ESM), Estrato socioeconómico medio-alto (ESA).

\section{DISCUSIÓN}

Los resultados del estudio muestran que uno de cada cuatro preescolares presenta un IFP inferior al promedio, además se observaron diferencias entre los IFP de los estratos socioeconómicos, siendo menores en los ESB y ESA en comparación con el ESM. Un estudio similar realizado en México, validó la pertinencia de la prueba DFH en niños, su correlación con la capacidad intelectual y su utilidad en la estimación de la madurez cognitiva (Fernández-Nistal, et al., 2018). Otros estudios muestran resultados similares tanto en la prevalencia como en cuanto al impacto del factor económico, social y cultural en el deterioro del desarrollo cognitivo y la influencia del ambiente familiar involucrada en la calidad de vida e inmadurez cognitiva del infante (El-Shafie, et al., 2019; Bolaños, et al., 2015; Mazzoni, et al., 2014; Solano, et al., 2017; García-Fernández, et al., 2018). En la actualidad, las cifras en México de niños con desarrollo atípico y con disminución en dos o más áreas del desarrollo son significativas (García-Fernández, et al., 2018), aunado a esta situación se considera que los niños presentan mayor susceptibilidad de padecer dificultades en las áreas relacionadas con el aprendizaje y, por lo tanto, de presentar un bajo rendimiento académico (Rizzoli-Córdoba, et al., 2015), lo que se 
relaciona con los pobres resultados en la prueba de DFH de determinados sectores infantiles.

Uno de los factores que predispone a los preescolares de ESB a presentar cifras menores del IFP son la desnutrición (Mamani-Ortiz, et al., 2014), la presencia de hogares disfuncionales, la violencia y la agresividad, lo que genera en más del $85 \%$ de los niños mala calidad de vida y un impacto negativo en su desenvolvimiento diario, así como el desarrollo de ansiedad y depresión (Herrera-Mora et al., 2019; Mazzoni, et al., 2014) que podrían verse reflejados en las pruebas proyectivas como la DFH. Los fenómenos anteriores se han comprobado mediante técnicas de neuroimagen donde se ha observado que, durante el período preescolar, el desarrollo cerebral de las regiones del lenguaje, las funciones ejecutivas y la memoria son sensibles a los efectos perjudiciales de la pobreza y las experiencias adversas en la infancia; por lo tanto, las condiciones socioeconómicas, ambientales y familiares desempeñan un fundamental papel en el desarrollo neurológico de los niños (Noble, et al., 2015). Respecto al bajo IFP observado en algunos preescolares que asisten a instituciones privadas, creemos que además de algunos factores de riesgo que comparten con los niños de ESB, podría existir la presencia de casos de sobreprotección en el ambiente familiar, lo que genera eventos negativos en el desarrollo psicosocial de los niños, quienes muestran disminución en la auto-suficiencia, dificultad para ajustarse al ambiente escolar, decremento en la confianza y autonomía e incapacidad de desenvolverse socialmente, lo que podría explicar el IFP por debajo del promedio (Schiffrin, \& Liss, 2017).

En este estudio, se observó un mayor IFP con el aumento de la edad y el grado escolar, que podría deberse a que los niños a mayor edad adquieren mayores competencias en relación con el conocimiento, inteligencia, lenguaje y aprendizaje. Asimismo, a partir del tercer año de preescolar y hasta finalizar el sexto año de primaria, los niños presentan el desarrollo de la motricidad fina sobre los movimientos voluntarios controlados por las manos y los dedos (Campo Ternera, 2009), situación que se observa con el progreso del desarrollo, la promoción del pensamiento, la resolución de problemas y el establecimiento de relaciones interpersonales, que se ven reflejadas en un mayor desempeño cognitivo (de Castro, et al., 2019). En contraparte, los niños de menor edad, quienes presentaron menores puntuaciones en el IFP, posiblemente transitaban por una fase de adaptación, experimentando desapego parental e inhibición conductual ante la ansiedad generada por 
estímulos desconocidos y frente a una menor experiencia de realizar tareas similares, que se manifiesta en un menor desempeño en la elaboración del DFH. Aunado a lo anterior, no hay que descartar la falta y/o carencia en la calidad de estimulación sensorial y motora, en los hogares previo al ingreso a la educación preescolar, las cuales pueden estar influyendo en los puntajes bajos del IFP en este grupo etario y evolucionar de acuerdo a edad y el desarrollo durante los años subsecuentes.

Por otro lado, generalmente se asocia al sexo femenino con un desarrollo precoz y un mayor IFP (Picard, 2015), en este estudio se observaron mayores cifras en el sexo masculino, esto podría deberse a factores psicosociales específicos de la población, ya que el desarrollo de las funciones psicológicas superiores está relacionado con el desarrollo madurativo del cerebro, la educación, la estimulación, las experiencias y las oportunidades de aprendizaje que, de acuerdo con las diversas poblaciones estudiadas, pueden variar. Otro factor que pudiera influir, son las características de la crianza de la región, que tienden a la sobreprotección del sexo femenino, lo que pudiera generar un déficit en la independencia, y repercutir en su IFP, además de posibles efectos emocionales y psicosociales durante su desarrollo (Masud, et al., 2019).

Similar a lo reportado en otros estudios, pudimos corroborar que factores como el número de hermanos, el orden de nacimiento, el tiempo de uso de la tecnología, realización de deporte y el grado de estudios de los padres no influyen en el IFP ni varían entre los estratos socioeconómicos, por lo que es necesario realizar más estudios que determinen los factores dentro de estos estratos, los estilos de vida y los ambientes escolares que intervienen o promueven un aumento o disminución del IFP, ya que estos podrían generar acciones para disminuir el RGD o políticas para incrementar el neurodesarrollo de los preescolares (Tükel, et al., 2018).

Infortunadamente, a pesar de tener identificados muchos de estos factores, no se han desarrollado estrategias adecuadas, ni políticas para contrarrestar y mejorar esta situación, motivo por el cual, estudios como el nuestro establecen una guía para la mejora de estas deficiencias (de Castro, et al., 2019). En México, a pesar de conocer los beneficios de la detección temprana de problemas del desarrollo regulados por las normas oficiales NOM031-SSA2 (Secretaria de Salud, 2011), en la cual se incluye la vigilancia del desarrollo de los niños menores de 5 años de edad, estas se aplican muy limitadamente. Basado en nuestros hallazgos, enfatizamos la necesidad de diseñar, así como de poner en 
funcionamiento estrategias y políticas para la elaboración de un tamizaje del desarrollo cognitivo en poblaciones infantiles durante los primeros años de vida como parte de la atención primaria a la salud, por medio de la aplicación de pruebas proyectivas como medida imprescindible desde el inicio de la educación preescolar, dadas las implicaciones que un diagnóstico tardío podría conllevar. Por otra parte, proponemos la utilización de estimaciones como la prueba del DFH, para la detección oportuna de preescolares en riesgo, dentro de las instituciones educativas.

Al mismo tiempo, resaltamos que gracias a la implementación de estas pruebas se podría reducir la prevalencia de problemas de salud mental durante la niñez y la adolescencia, así como la susceptibilidad a psicopatologías y abuso de drogas (Bornstein, et al., 2013). También enfatizamos el establecimiento de mejores estrategias de acercamiento con padres de familia, docentes y directivos de los preescolares, para concientizar sobre la importancia de la correcta estimulación sensorial, principalmente en la motricidad gruesa a través de la educación física y/o el ejercicio (Merrick, et al., 2017), así como la estimulación de las funciones mentales superiores (autocuidado, independencia, empatía, memoria, atención y funciones ejecutivas), y la reducción de la sobreprotección y las horas de uso de las tecnologías. Por lo tanto, el seguir omitiendo este tipo de pruebas previo al ingreso y durante los continuos años de formación inicial, generaran que las prevalencias de niños con carencias en el desarrollo continúen aumentando.

Las debilidades de este estudio incluyen la falta de medición de otras variables que pueden aumentar o disminuir el IFP, como la estimulación, disfunción en el hogar, malnutrición, violencia, agresividad y estado emocional. Las fortalezas incluyen un tamaño de muestra considerable, la accesibilidad y versatilidad de la prueba, así como la inclusión de preescolares sanos y sin características especiales, situación poco frecuente en estudios similares, cuyas poblaciones de estudio son específicas y particulares. No se reportaron conflictos de interés por parte de ninguno de los autores. Por otro parte, la selección del instrumento (la prueba del DFH) utilizado durante el presente estudio, se basó principalmente en la factibilidad y accesibilidad hacia la muestra seleccionada, este instrumento ya ha sido ampliamente utilizado y validado para distintas poblaciones, en las que en algunos casos se ha demostrado su correlación con otras pruebas diagnósticas más complejas (Springer, et al., 2020; Zeegers, et al., 2009; Hamilton, 2006; Baraheni, et al., 2018; Rinaldi, \& Alves, 2014; Fernández-Nistal, et al., 2018; Senol, \& Ochilbek, 
2020; Piotrowski, 2015; Troncone, et al., 2021). No obstante, estamos conscientes de las limitantes propias de la prueba del DFH, sin embargo, para las finalidades de este estudio, aunado a la necesidad de estimación del desarrollo hacia otras poblaciones similares en otras regiones, consideramos que esta prueba es adecuada.

En conclusión, en la población estudiada, uno de cada cuatro preescolares presenta un nivel de desarrollo por debajo de lo esperado para su edad, siendo los preescolares de ESM los que presentan mayores puntajes del IPF. Asimismo, se detectó que el IFP tiene relación el sexo masculino, mayores edades y grado escolar. Además, resaltamos que existe una importante necesidad de implementar programas de capacitación para promover el desarrollo de la motricidad gruesa y las funciones mentales superiores dentro del hogar y en las escuelas, con el objetivo de proveer de estímulos positivos del en el desarrollo de los preescolares. En la actualidad, es apremiante la mejora de una cultura de vigilancia continua del desarrollo cognitivo dentro de las instituciones educativas, que permita ubicar a los preescolares en riesgo y que estos puedan obtener un diagnóstico presuntivo precoz y tratamiento oportuno, para asegurar que puedan alcanzar su potencial, así como mejorar la calidad de vida de los niños y de sus familias.

\section{REFERENCIAS BIBLIOGRAFICAS}

Baraheni, N., Heidarabady, S., Nemati, S., y Ghojazadeh, M. (2018). Goodenough-Harris Drawing a Man Test (GHDAMT) as a Substitute of Ages and Stages Questionnaires (ASQ2) for Evaluation of Cognition. Iranian journal of child neurology, 12(4), 94-102.

Black, M. M., Walker, S. P., Fernald, L., Andersen, C. T., DiGirolamo, A. M., Lu, C., McCoy, D. C., Fink, G., Shawar, Y. R., Shiffman, J., Devercelli, A. E., Wodon, Q. T., Vargas-Barón, E., Grantham-McGregor, S., y Lancet Early Childhood Development Series Steering Committee (2017). Early childhood development coming of age: science through the life course. Lancet (London, England), 389(10064), 77-90. https://doi.org/10.1016/S0140-6736(16)31389-7

Bolaños, C., Mina-Lara, M. del M., y Ramos-Ortega, G. (2015). Indicadores de riesgo del Perfil de Conductas de Desarrollo como instrumento para la detección temprana de retrasos y alteraciones del desarrollo. Acta pediátrica de México, 36(3), 135-146. 
Bornstein, M. H., Hahn, C. S., y Suwalsky, J. T. (2013). Developmental Pathways among Adaptive Functioning and Externalizing and Internalizing Behavioral Problems: Cascades from Childhood into Adolescence. Applied developmental science, 17(2), 76-87. https://doi.org/10.1080/10888691.2013.774875

Campo Ternera, L. A. (2009). Características del desarrollo cognitivo y del lenguaje en niños de edad preescolar. Psicogente, 12(22). http://revistas.unisimon.edu.co/index.php/psicogente/article/view/1168

Clark, H., Coll-Seck, A., Banerjee, A., Peterson, S., Dalglish, S., y Ameratunga, S. et al. (2020). A future for the world's children? A WHO-UNICEF-Lancet Commission. The Lancet, 395(10224), 605-658. https://doi.org/10.1016/S01406736(19)32540-1

Daelmans, B., Darmstadt, G., Lombardi, J., Black, M., Britto, P., y Lye, S. et al. (2017). Early childhood development: the foundation of sustainable development. The Lancet, 389(10064), 9-11. https://doi.org/10.1016/S0140-6736(16)31659-2

de Castro, F., Vázquez-Salas, R. A., Villalobos, A., Rubio-Codina, M., Prado, E., Sánchez-Ferrer, J. C., Romero, M., y Shamah-Levy, T. (2019). Contexto y resultados del desarrollo infantil temprano en niños y niñas de 12 a 59 meses en México. Salud Pública De México, 61(6), 775-786. https://doi.org/10.21149/10560

El-Shafie AM, El Lahony DM, Abd El Latif ZO, Khalil MO. (2019). Draw-a-person test as a tool for intelligence screening in primary school children. Menoufia Medical Journal, 2019;32:329-34.

Engle, P. L., Black, M. M., Behrman, J. R., Cabral de Mello, M., Gertler, P. J., Kapiriri, L., Martorell, R., Young, M. E., y International Child Development Steering Group (2007). Strategies to avoid the loss of developmental potential in more than 200 million children in the developing world. Lancet (London, England), 369(9557), 229-242. https://doi.org/10.1016/S0140-6736(07)60112-3

Fernández-Nistal, M. T., Ponce-Zaragoza, F. A., y Ochoa-Ávila, E. (2018). La Validez del Dibujo de la Figura Humana como una Medida de la Madurez Intelectual en Niños Indígenas Yaquis. Revista Iberoamericana de Diagnóstico y Evaluación - e Avaliação Psicológica, 3(48), 41-52. 
García-Fernández, D. A., Velázquez-Saucedo, G., Guedea-Delgado, J. C., Sías-Casas, M., y Zubiaur-González, M. (2018). Indicadores de riesgo del rendimiento escolar en niños de 3 a 6 años. TECNOCIENCIA Chihuahua, 11(3), 115-126.

Gridley, N., Blower, S., Dunn, A., Bywater, T., y Bryant, M. (2019). Psychometric Properties of Child (0-5 Years) Outcome Measures as used in Randomized Controlled Trials of Parent Programs: A Systematic Review. Clinical Child And Family Psychology Review, 22(3), 388-405. https://doi.org/10.1007/s10567-01900277-1

Gutiérrez Duarte, S. A., y Ruiz León, M. (2018). Impacto de la educación inicial y preescolar en el neurodesarrollo infantil. IE Revista de investigación educativa de la REDIECH, 9(17), 33-51.

Hamilton, S. (2006). Screening for developmental delay: reliable, easy-to-use tools. The Journal of family practice, 55(5), 415-422.

Herrera-Mora, D. B., Munar-Torres, Y. E., Molina-Achury, N. J., y Robayo-Torres, A. L. (2019). Desarrollo infantil y condición socioeconómica. Artículo de revisión. Revista de la Facultad de Medicina, 67(1), 145-152. https://doi.org/10.15446/revfacmed.v67n1.66645

Lebel, C. y Deoni, S. (2018). The development of brain white matter microstructure. NeuroImage, 182, 207-218. https://doi.org/10.1016/j.neuroimage.2017.12.097

Mamani Ortiz, Y., Choque Ontiveros, M. C., y Rojas Salazar, E. G. (2014). Estado nutricional y su relación con el coeficiente intelectual de niños en edad escolar. Gaceta Médica Boliviana, 37(1), 6-10.

Masud, S., Mufarrih, S., Qureshi, N., Khan, F., Khan, S., y Khan, M. (2019). Academic Performance in Adolescent Students: The Role of Parenting Styles and SocioDemographic Factors - A Cross Sectional Study From Peshawar, Pakistan. Frontiers In Psychology, 10. https://doi.org/10.3389/fpsyg.2019.02497

Mazzoni, C. C., Stelzer, F., Cervigni, M. A., y Martino, P. (2014). Impacto de la pobreza en el desarrollo cognitivo: un análisis teórico de dos factores mediadores. Liberabit, 20(1), 93-100. Recuperado en 02 de septiembre de 2021, de http://www.scielo.org.pe/scielo.php?script=sci_arttext\&pid=S1729$\underline{48272014000100008 \& \operatorname{lng}=e s \& t \operatorname{lng}=e s}$. 
Merrick, M. T., Ports, K. A., Ford, D. C., Afifi, T. O., Gershoff, E. T., y Grogan-Kaylor, A. (2017). Unpacking the impact of adverse childhood experiences on adult mental health. Child abuse \& neglect, 69, 10-19. https://doi.org/10.1016/j.chiabu.2017.03.016

Myers, Robert G.. (2015). Desarrollo infantil temprano en México: avances y retos. Boletín médico del Hospital Infantil de México, 72(6), 359-361. https://doi.org/10.1016/j.bmhimx.2015.11.001

Noble, K. G., Houston, S. M., Brito, N. H., Bartsch, H., Kan, E., Kuperman, J. M., Akshoomoff, N., et al., (2015). Family income, parental education and brain structure in children and adolescents. Nature neuroscience, 18(5), 773-778. https://doi.org/10.1038/nn.3983

Picard, D. (2015). Sex Differences in Scores on the Draw-a-Person Test across Childhood: Do They Relate to Graphic Fluency?. Perceptual and Motor Skills, 120(1), 273-287. https://doi.org/10.2466/10.27.PMS.120v10x1

Piotrowski, C. (2015). Projective techniques usage worldwide: A review of applied settings 1995-2015. Journal of the Indian Academy of Applied Psychology, 41(3), 9-19.

Ramírez Montenegro, J., Valencia Valencia, D., y Parra Martínez, N. (2014). La escala de Goodenough-Harris y su utilidad en la detección de discapacidad cognitiva en una población de niños de 3 a 13 años. Revista Colombiana De Médicina Física y Rehabilitación, 24(1), 27-36. https://doi.org/10.28957/rcmfr.v24n1a2

Rinaldi, H. R., y Alves, I. C. B. (2014). Estudo normativo do Teste Goodenough-Harris em crianças na cidade de São Paulo. Boletim - Academia Paulista de Psicologia, 34(87), 336-351.

http://pepsic.bvsalud.org/scielo.php?script=sci_arttext\&pid=S1415$\underline{711 X 2014000200004 \& \operatorname{lng}=\text { es\&tlng=pt. }}$

Rizzoli-Córdoba, A., Campos-Maldonado, M. C., Vélez-Andrade, V. H., DelgadoGinebra, I., Baqueiro-Hernández, C. I., Villasís-Keever, M. Á., et al., (2015). Evaluación diagnóstica del nivel de desarrollo en niños identificados con riesgo de retraso mediante la prueba de Evaluación del Desarrollo Infantil. Boletín médico del Hospital Infantil de México, 72(6), 397-408. https://doi.org/10.1016/j.bmhimx.2015.11.005 
Sánchez-Villena, A. R., y Ventura-León, J. (2020). Pruebas proyectivas: ¿Son útiles para la evaluación infantil?. Revista chilena de pediatría, 91(3), 466. https://dx.doi.org/10.32641/rchped.v91i3.2017

Schiffrin, H., y Liss, M. (2017). The Effects of Helicopter Parenting on Academic Motivation. Journal Of Child And Family Studies, 26(5), 1472-1480. https://doi.org/10.1007/s10826-017-0658-z

Secretaria de Salud. (2001). Norma Oficial Mexicana, NOM-031- SSA2-1999 para la atención a la salud del niño 1999. México; Feb, 2001, [en línea] disponible en: http://www.salud.gob.mx/unidades/cdi/nom/031ssa29.html

SEFIPLAN. (2018). Secretaria de Finanzas y planeación, sistema de información Municipal, Municipio de Zaragoza, [en línea] disponible en http://ceieg.veracruz.gob.mx/wp-content/uploads/sites/21/2018/05/Zaragoza.pdf. SEFIPLAN. (2020). Secretaria de Finanzas y planeación, Sistema de Información Municipal, Municipio de Minatitlán, [en línea] disponible en http://ceieg.veracruz.gob.mx/wpcontent/uploads/sites/21/2020/12/Minatitlán_2020.pdf

Senol, D., y Ochilbek, R. (2020). Effect of the Age and Gender on the Reliability of Draw-a-Person Test. Journal Of Research In Medical And Dental Science, 8(5), 151-158.

Solano, F. E., Vilela-Estrada, M. A, Meza-Liviapo, J., Araujo-Chumacero, M. M., Vilela-Estrada, A. L., y Mejia, C. R. (2017). Factores sociofamiliares asociados a la calidad de vida en niños de colegios de la ciudad de Piura, Perú. Revista chilena de pediatría, 88(2), 223-229. http://dx.doi.org/10.1016/j.rchipe.2016.07.012.

Springer, P. E., Kalk, E., Pretorius, C., Chirehwa, M. T., Kruger, M., Cotton, M. F., y Laughton, B. (2020). Value of the Goodenough Drawing Test as a research tool to detect developmental delay in South African preschool children. South African Journal of Psychology, 50(1), 81-91. https://doi.org/10.1177/0081246319850683 Troncone, A., Chianese, A., Di Leva, A. et al. (2021). Validity of the Draw a Person: A Quantitative Scoring System (DAP:QSS) for Clinically Evaluating Intelligence. Child Psychiatry \& Human Development. 52, 728-738. https://doi.org/10.1007/s10578-020-01058-6 
Tükel, Ş., Eliasson, A., Böhm, B., y Smedler, A. (2018). Simple Categorization of Human Figure Drawings at 5 Years of Age as an Indicator of Developmental Delay. Developmental Neurorehabilitation, 22(7), 479-486. https://doi.org/10.1080/17518423.2018.1532969

Vitrikas, K., Savard, D., y Bucaj, M. (2017). Developmental Delay: When and How to Screen. American family physician, 96(1), 36-43.

Zeegers, I., Rabie, H., Swanevelder, S., Edson, C., Cotton, M., y van Toorn, R. (2009). Attention Deficit Hyperactivity and Oppositional Defiance Disorder in HIVInfected South African Children. Journal Of Tropical Pediatrics, 56(2), 97-102. https://doi.org/10.1093/tropej/fmp072 